\title{
Anomalous dimensions at five loops
}

\section{Thomas Luthe}

Faculty of Physics, University of Bielefeld,

33501 Bielefeld, Germany

\section{Andreas Maier*}

Institute for Particle Physics Phenomenology, Durham University,

Durham, United Kingdom

E-mail: andreas.maier@durham.ac.uk

\section{Peter Marquard}

Deutsches Elektronen Synchrotron (DESY),

Platanenallee 6, Zeuthen, Germany

\section{York Schröder}

Grupo de Cosmología y Partículas Elementales, Universidad del Bío-Bío,

Casilla 447, Chillán, Chile

We discuss the recent calculation of the five-loop Beta function and the full set of the renormalization constants of QCD up to the linear term in the gauge parameter $\xi=1-\xi_{L}$.

13th International Symposium on Radiative Corrections (Applications of Quantum Field Theory to Phenomenology)

25-29 September, 2017

St. Gilgen, Austria

${ }^{*}$ Speaker. 


\section{Introduction}

The features of a gauge theory are to a large extent described by their anomalous dimensions. In Quantum Chromodynamics (QCD), the most important ones are the Beta function and the anomalous mass dimension $\gamma_{m}$, which together govern the running of the strong coupling constant and the heavy quark mass. In addition to these gauge-independent physical anomalous dimensions, the complete renormalization of QCD at a given loop order requires the knowledge of at least three more unphysical anomalous dimensions. Here, we choose the anomalous dimensions of the quark fields $\gamma_{2}$, the gluon field $\gamma_{3}$, and the ghost-gluon vertex $\gamma_{1}^{c c g}$. All other anomalous dimensions can then be derived via Ward identities. The anomalous dimensions are related to the corresponding renormalization constants by

$$
\gamma_{i}=-\partial_{\ln \mu^{2}} \ln Z_{i}
$$

Anomalous dimensions have been studied to a large extent over the last decades. The Beta function was first discussed in the groundbreaking works [1,2] establishing QCD and demonstrating its asymptotic freedom. Perturbative corrections have then been pushed to two-loop [3, 4], three-loop [5, 6] and four-loop [7, 8] level. Five-loop results have appeared over the last ten years or so, first for the case of Quantum Electrodynamics (QED) [9, 10, 11], then for physical QCD with gauge group SU(3) [12, 13], and finally for a generalization of QCD to arbitrary simple gauge (Lie) groups [14, 15, 18, 19].

The second most important representative, the (gauge-invariant) anomalous dimension of the quark mass, has been known at two [20] and three loops [21, 22] for a long time already; at four loops, complete results for $\mathrm{SU}(N)$ and QED as well as general Lie groups are available [23, 24]; at five loops, mass renormalization is known for $\mathrm{SU}(3)$ as well as general Lie groups $[16,25,26]$.

The remaining members of the set of anomalous dimensions depend on the gauge parameter. At four loops, these are known since more than a decade for $\mathrm{SU}(N)$ and general Lie groups, see $[8,27]$ and references therein. Full gauge dependence for the case of a general Lie group has been added only recently $[16,17]$. At five loops and for a general Lie group, all of them are known in Feynman gauge from $[16,17]$. The linear dependence on the gauge parameter has been calculated in [18] and the full gauge dependence in [19].

In the following, we briefly review the calculation performed in [18] presenting our results for the Beta function and the ghost field anomalous dimension $\gamma_{3}^{c}=\gamma_{1}^{c c g}-\frac{\gamma_{3}}{2}-\frac{\beta}{2}$ and visualize the five-loop effects on the running of the strong coupling constant.

\section{Computation and Results}

In our calculation we followed the approach suggested in [28, 29] employing fully massive vacuum integrals to eliminate infrared divergences. This approach is different from the methods used in other calculations of the five-loop renormalization constants $[9,10,11,26,12,13,15$, $25,19]$ and thus provides a completely independent result. As examples, we show our results for the Beta function and the ghost field anomalous dimension. For details of the calculation and a complete list of results we refer the reader to [18]. 
In order to be able to present our results we first need to define our notation concerning group invariants. We use the same notation that has already been utilized in our previous works [14, 16, 17]. We focus on a Yang-Mills theory coupled to $N_{\mathrm{f}}$ fermions in the fundamental representation. It is straightforward to generalize our results to fermions in a (single) arbitrary representation $R$ by substituting all generators of the fundamental representation with generators of $R$.

We use $C_{\mathrm{A}}$ and $C_{\mathrm{F}}$ to denote the eigenvalues of the Casimir operators in the adjoint and fundamental representation (of dimensions $N_{\mathrm{F}}$ and $N_{\mathrm{A}}$, respectively) as usual. $T_{\mathrm{F}}$ is the index of the fundamental representation and $N_{\mathrm{f}}$ denotes the number of fermions. To facilitate compact representations of our results, we find it convenient to use the following normalized combinations of group invariants:

$$
n_{f}=\frac{N_{\mathrm{f}} T_{\mathrm{F}}}{C_{\mathrm{A}}} \quad, \quad c_{f}=\frac{C_{\mathrm{F}}}{C_{\mathrm{A}}} .
$$

In loop diagrams, one typically encounters traces of more than two group generators, giving rise to higher-order group invariants. These higher-order traces can be systematically classified in terms of combinations of symmetric tensors [30]. Rewriting the generators of the adjoint representation as $\left[F^{a}\right]_{b c}=-i f^{a b c}$, we need the following three combinations (again, we normalize conveniently):

$$
\begin{aligned}
& d_{1}=\frac{\left[\mathrm{s} \operatorname{Tr}\left(T^{a} T^{b} T^{c} T^{d}\right)\right]^{2}}{N_{\mathrm{A}} T_{\mathrm{F}}^{2} C_{\mathrm{A}}^{2}}, d_{2}=\frac{\mathrm{s} \operatorname{Tr}\left(T^{a} T^{b} T^{c} T^{d}\right) \mathrm{s} \operatorname{Tr}\left(F^{a} F^{b} F^{c} F^{d}\right)}{N_{\mathrm{A}} T_{\mathrm{F}} C_{\mathrm{A}}^{3}} \\
& d_{3}=\frac{\left[\mathrm{s} \operatorname{Tr}\left(F^{a} F^{b} F^{c} F^{d}\right)\right]^{2}}{N_{\mathrm{A}} C_{\mathrm{A}}^{4}}
\end{aligned}
$$

Here, $\operatorname{sir}(A B C D)=\frac{1}{6} \operatorname{Tr}(A B C D+A B D C+A C B D+A C D B+A D B C+A D C B)$ is a fully symmetrized trace.

Specializing to the gauge group $\mathrm{SU}(N)$, thus setting $T_{\mathrm{F}}=\frac{1}{2}$ and $C_{\mathrm{A}}=N$, our set of normalized invariants reads [30]

$$
\begin{aligned}
& n_{f}=\frac{N_{\mathrm{f}}}{2 N}, c_{f}=\frac{N^{2}-1}{2 N^{2}}, \\
& d_{1}=\frac{N^{4}-6 N^{2}+18}{24 N^{4}}, d_{2}=\frac{N^{2}+6}{24 N^{2}}, d_{3}=\frac{N^{2}+36}{24 N^{2}} .
\end{aligned}
$$

From here, one can for example easily obtain the SU(3) coefficients, corresponding to physical QCD.

For the Beta function defined through

$$
\partial_{\ln \mu^{2}} a=-a[\varepsilon-\beta]=-a\left[\varepsilon+b_{0} a+b_{1} a^{2}+b_{2} a^{3}+b_{3} a^{4}+b_{4} a^{5}+\ldots\right],
$$

with

$$
a \equiv \frac{C_{A} \alpha_{s}}{4 \pi}=\frac{C_{A} g^{2}}{16 \pi^{2}}
$$


we obtained

$$
\begin{aligned}
& 3^{1} b_{0}= {[-4] n_{f}+11 } \\
& 3^{5} b_{4}= b_{44} n_{f}^{4}+b_{43} n_{f}^{3}+b_{42} n_{f}^{2}+b_{41} n_{f}+b_{40} \\
& b_{44}=\left\{c_{f}, 1\right\} \cdot\left\{-8\left(107+144 \zeta_{3}\right), 4\left(229-480 \zeta_{3}\right)\right\} \\
& b_{43}=\left\{c_{f}^{2}, c_{f}, d_{1}, 1\right\} \cdot\left\{-6\left(4961-11424 \zeta_{3}+4752 \zeta_{4}\right)\right. \\
&-48\left(46+1065 \zeta_{3}-378 \zeta_{4}\right), 1728\left(55-123 \zeta_{3}+36 \zeta_{4}+60 \zeta_{5}\right) \\
&\left.-3\left(6231+9736 \zeta_{3}-3024 \zeta_{4}-2880 \zeta_{5}\right)\right\} \\
& b_{42}=\left\{c_{f}^{3}, c_{f}^{2}, c_{f} d_{1}, c_{f}, d_{2}, d_{1}, 1\right\} \cdot\left\{-54\left(2509+3216 \zeta_{3}-6960 \zeta_{5}\right)\right. \\
& 9\left(94749 / 2-28628 \zeta_{3}+10296 \zeta_{4}-39600 \zeta_{5}\right) \\
& 25920\left(13+16 \zeta_{3}-40 \zeta_{5}\right), 3\left(5701 / 2+79356 \zeta_{3}-25488 \zeta_{4}+43200 \zeta_{5}\right) \\
&-864\left(115-1255 \zeta_{3}+234 \zeta_{4}+40 \zeta_{5}\right) \\
&-432\left(1347-2521 \zeta_{3}+396 \zeta_{4}-140 \zeta_{5}\right) \\
&\left.843067 / 2+166014 \zeta_{3}-8424 \zeta_{4}-178200 \zeta_{5}\right\} \\
& b_{41}=\left\{c_{f}^{4}, c_{f}^{3}, c_{f}^{2}, c_{f} d_{2}, c_{f}, d_{3}, d_{2}, 1\right\} \cdot\left\{-81\left(4157 / 2+384 \zeta_{3}\right)\right. \\
& 81\left(11151+5696 \zeta_{3}-7480 \zeta_{5}\right) \\
&-3\left(548732+151743 \zeta_{3}+13068 \zeta_{4}-346140 \zeta_{5}\right) \\
&-25920\left(3-4 \zeta_{3}-20 \zeta_{5}\right) \\
& 8141995 / 8+35478 \zeta_{3}+73062 \zeta_{4}-706320 \zeta_{5} \\
& 216\left(113-2594 \zeta_{3}+396 \zeta_{4}+500 \zeta_{5}\right) \\
& 216\left(1414-15967 \zeta_{3}+2574 \zeta_{4}+8440 \zeta_{5}\right) \\
&\left.-5048959 / 4+31515 \zeta_{3}-47223 \zeta_{4}+298890 \zeta_{5}\right\} \\
&\left.8296235 / 16-4890 \zeta_{3}+9801 \zeta_{4} / 2-28215 \zeta_{5}\right\} \\
&\left.b_{40}, 1\right\} \cdot\left\{-162\left(257-9358 \zeta_{3}+1452 \zeta_{4}+7700 \zeta_{5}\right)\right. \\
&
\end{aligned}
$$

where we use a scalar-product-like notation. We have only listed the one-loop result $b_{0}$ for normalization and the new five-loop result $b_{4}$.

As a further example we show our result for the linearly gauge-parameter dependent part of $\gamma_{3}^{c}$ at five loops

$$
\gamma_{3}^{c}=-a\left[-\frac{1}{4}(2+\xi)+\gamma_{31}^{c} a+\gamma_{32}^{c} a^{2}+\gamma_{33}^{c} a^{3}+\gamma_{34}^{c} a^{4}+\ldots\right]
$$




$$
\begin{aligned}
2^{14} 3^{5} \gamma_{34}^{c}= & \gamma_{344}^{c}\left[16 n_{f}\right]^{4}+\gamma_{343}^{c}\left[16 n_{f}\right]^{3}+\gamma_{342}^{c}\left[16 n_{f}\right]^{2}+\gamma_{341}^{c}\left[16 n_{f}\right]+\gamma_{340}^{c} \\
\gamma_{34 i}^{c}= & \gamma_{34 i 0}^{c}+\xi \gamma_{34 i 1}^{c}+\mathscr{O}\left(\xi^{2}\right) \\
\gamma_{3441}^{c}= & 0 \\
\gamma_{3431}^{c}= & \left\{c_{f}, 1\right\} \cdot\left\{0,2\left(569+576 \zeta_{3}-1296 \zeta_{4}\right)\right\} \\
\gamma_{3421}^{c}= & \left\{c_{f}^{2}, c_{f}, d_{1}, d_{2}, 1\right\} \cdot\left\{0,36\left(-8191+6984 \zeta_{3}+1944 \zeta_{4}-3456 \zeta_{5}\right), 0,0\right. \\
& \left.-2\left(66745+295182 \zeta_{3}-23328 \zeta_{4}-208764 \zeta_{5}\right)\right\} \\
\gamma_{3411}^{c}= & \left\{c_{f}^{3}, c_{f}^{2}, c_{f} d_{2}, c_{f}, d_{2}, d_{3}, 1\right\} \cdot\left\{0,-5184\left(1349+3018 \zeta_{3}-720 \zeta_{3}^{2}+666 \zeta_{4}\right.\right. \\
& \left.-2520 \zeta_{5}-1800 \zeta_{6}\right), \\
& 0,144\left(90827+34092 \zeta_{3}+7776 \zeta_{3}^{2}-15552 \zeta_{4}-32832 \zeta_{5}-32400 \zeta_{6}\right) \\
& 5184\left(32+4008 \zeta_{3}+432 \zeta_{4}-3060 \zeta_{5}-900 \zeta_{6}-1323 \zeta_{7}\right) \\
& 2592\left(208-1141 \zeta_{3}+162 \zeta_{3}^{2}-297 \zeta_{4}-8375 \zeta_{5}+3525 \zeta_{6}+882 \zeta_{7}\right) \\
& 4\left(3979604+2404521 \zeta_{3}-750222 \zeta_{3}^{2}+1808649 \zeta_{4}-4632336 \zeta_{5}\right. \\
& \left.\left.-1111725 \zeta_{6}+904932 \zeta_{7}\right)\right\} \\
\gamma_{3401}^{c}= & \left\{d_{3}, 1\right\} \cdot\left\{1 0 3 6 8 \left(2732-13091 \zeta_{3}-4146 \zeta_{3}^{2}-2241 \zeta_{4}+150485 \zeta_{5}\right.\right. \\
& \left.-50925 \zeta_{6}-14434 \zeta_{7}\right), \\
& -144\left(55138033 / 36-72901 \zeta_{3}-105498 \zeta_{3}^{2}+1074645 \zeta_{4}-1516578 \zeta_{5}\right. \\
& \left.\left.-467775 \zeta_{6}+68397 \zeta_{7}\right)\right\} .
\end{aligned}
$$

We observe that only 10 of the 17 possible color structures contain terms linear in $\xi$.

To illustrate the effect of the five-loop effects on the evolution of the strong coupling constant we compare in Fig. 1 its running from $m_{\tau}$ to $m_{Z}$ using one-loop to five-loop approximation. We normalize to the four-loop result and include the $(N-1)$-loop decoupling of $\alpha_{s}$ at the bottom threshold. As can be seen from the slope of the lines, the difference between four- and five-loop evolution for a fixed number of flavors is very small. The final change in the value of $\alpha_{s}\left(m_{Z}\right)$ is dominated by the decoupling correction and comparable to the difference between the three- and four-loop results. However, these differences are negligible compared to the parametric uncertainty obtained from $\alpha_{s}\left(m_{\tau}\right)=0.325 \pm 0.015[31]$.

\section{Conclusions}

We presented results for the gauge invariant Beta function and the linear term in the gauge parameter dependence of the ghost wave function renormalization constant obtained in [18]. All our results agree with or have been confirmed in the literature. The numerical effects at five-loop order on the running of the strong coupling have been shown to be small.

\section{References}

[1] D. J. Gross and F. Wilczek, Phys. Rev. Lett. 30 (1973) 1343. 


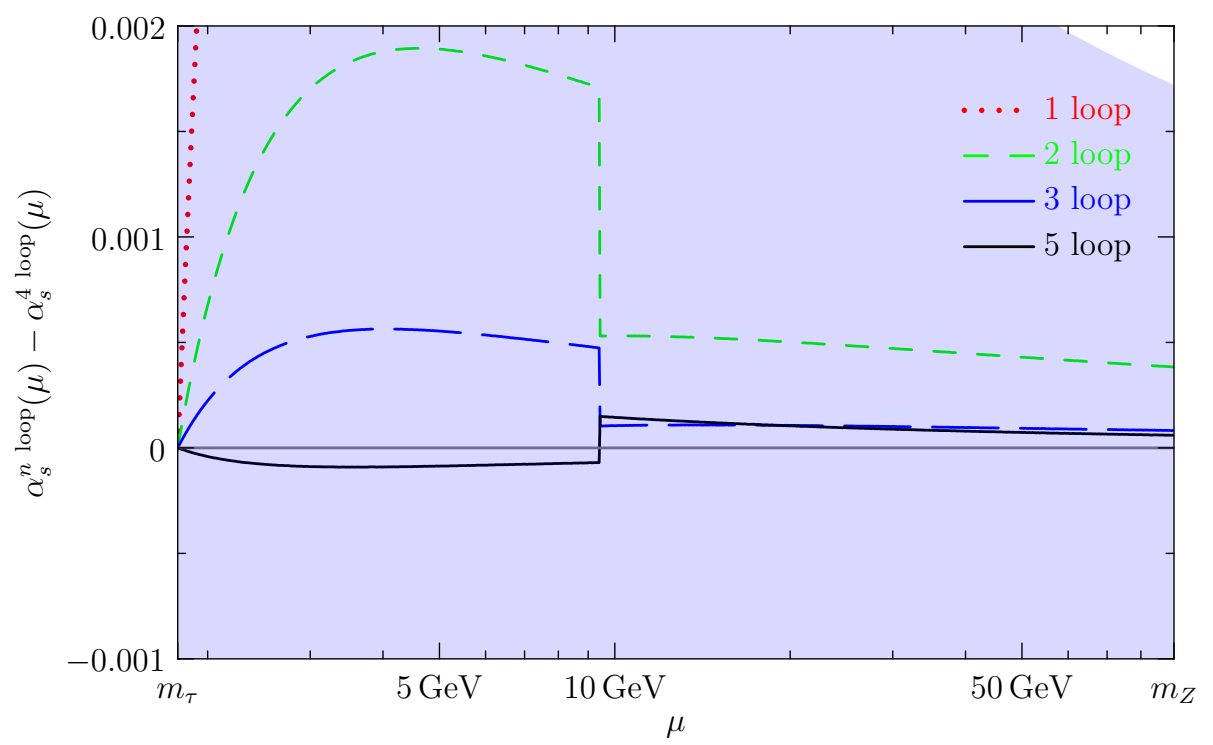

Figure 1: Running of the strong coupling constant $\alpha_{s}$ from $\mu=m_{\tau}$ to $\mu=m_{Z}$ including decoupling at the bottom threshold. The shaded area shows the parametric uncertainty propagated from the initial value $\alpha_{s}\left(m_{\tau}\right)=0.325 \pm 0.015$.

[2] H. D. Politzer, Phys. Rev. Lett. 30 (1973) 1346.

[3] W. E. Caswell, Phys. Rev. Lett. 33 (1974) 244.

[4] D. R. T. Jones, Nucl. Phys. B 75 (1974) 531.

[5] O. V. Tarasov, A. A. Vladimirov and A. Y. Zharkov, Phys. Lett. B 93 (1980) 429.

[6] S. A. Larin and J. A. M. Vermaseren, Phys. Lett. B 303 (1993) 334 [hep-ph/9302208].

[7] T. van Ritbergen, J. A. M. Vermaseren and S. A. Larin, Phys. Lett. B 400 (1997) 379 [hep-ph/9701390].

[8] M. Czakon, Nucl. Phys. B 710 (2005) 485 [hep-ph/0411261].

[9] P. A. Baikov, K. G. Chetyrkin and J. H. Kühn, PoS RADCOR 2007 (2007) 023 [arXiv:0810.4048].

[10] P. A. Baikov, K. G. Chetyrkin and J. H. Kühn, Phys. Rev. Lett. 104 (2010) 132004 [arXiv:1001.3606].

[11] P. A. Baikov, K. G. Chetyrkin, J. H. Kühn and J. Rittinger, JHEP 1207 (2012) 017 [arXiv:1206.1284].

[12] K. Chetyrkin, P. Baikov and J. Kühn, PoS LL 2016 (2016) 010.

[13] P. A. Baikov, K. G. Chetyrkin and J. H. Kühn, Phys. Rev. Lett. 118 (2017) no.8, 082002 [arXiv:1606.08659].

[14] T. Luthe, A. Maier, P. Marquard and Y. Schröder, JHEP 1607 (2016) 127 [arXiv:1606.08662].

[15] F. Herzog, B. Ruijl, T. Ueda, J. A. M. Vermaseren and A. Vogt, JHEP 1702 (2017) 090 [arXiv:1701.01404].

[16] T. Luthe, A. Maier, P. Marquard and Y. Schröder, JHEP 1701 (2017) 081 [arXiv:1612.05512].

[17] T. Luthe, A. Maier, P. Marquard and Y. Schröder, JHEP 1703 (2017) 020 [arXiv:1701.07068].

[18] T. Luthe, A. Maier, P. Marquard and Y. Schröder, JHEP 1710, 166 (2017) [arXiv:1709.07718]. 
[19] K. G. Chetyrkin, G. Falcioni, F. Herzog and J. A. M. Vermaseren, JHEP 1710, 179 (2017) Addendum: [JHEP 1712, 006 (2017)] [arXiv:1709.08541].

[20] R. Tarrach, Nucl. Phys. B 183 (1981) 384.

[21] O. V. Tarasov, JINR-P2-82-900 (in Russian).

[22] S. A. Larin, Phys. Lett. B 303 (1993) 113 [hep-ph/9302240].

[23] K. G. Chetyrkin, Phys. Lett. B 404 (1997) 161 [hep-ph/9703278].

[24] J. A. M. Vermaseren, S. A. Larin and T. van Ritbergen, Phys. Lett. B 405 (1997) 327 [hep-ph/9703284].

[25] P. A. Baikov, K. G. Chetyrkin and J. H. Kühn, JHEP 1704 (2017) 119 [arXiv:1702.01458].

[26] P. A. Baikov, K. G. Chetyrkin and J. H. Kühn, JHEP 1410 (2014) 076 [arXiv:1402.6611].

[27] K. G. Chetyrkin, Nucl. Phys. B 710 (2005) 499 [hep-ph/0405193].

[28] M. Misiak and M. Münz, Phys. Lett. B 344, 308 (1995) [hep-ph/9409454].

[29] K. G. Chetyrkin, M. Misiak and M. Münz, Nucl. Phys. B 518 (1998) 473 [hep-ph/9711266].

[30] T. van Ritbergen, A. N. Schellekens and J. A. M. Vermaseren, Int. J. Mod. Phys. A 14 (1999) 41 [hep-ph/9802376].

[31] C. Patrignani et al. [Particle Data Group], Chin. Phys. C 40, no. 10, 100001 (2016). 